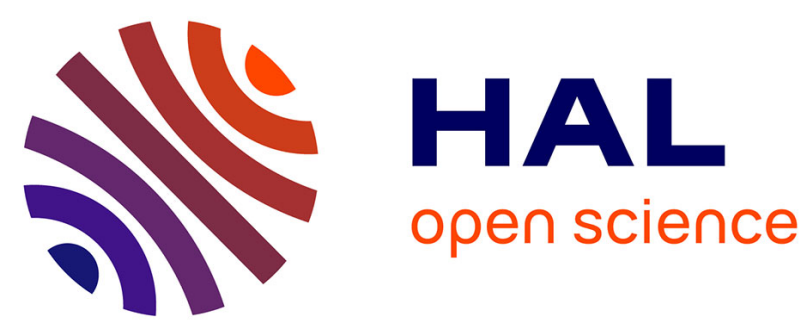

\title{
Geometric synthesis of a hybrid limit cycle for the stabilizing control of a class of nonlinear switched dynamical systems
}

\author{
J. Ben Salah, C. Valentin, H. Jerbi, C.Z. Xu
}

\section{- To cite this version:}

J. Ben Salah, C. Valentin, H. Jerbi, C.Z. Xu. Geometric synthesis of a hybrid limit cycle for the stabilizing control of a class of nonlinear switched dynamical systems. Systems and Control Letters, 2011, 60 (12), pp.967 - 976. 10.1016/j.sysconle.2011.08.005 . hal-01882655

\section{HAL Id: hal-01882655 \\ https://hal.science/hal-01882655}

Submitted on 27 Nov 2019

HAL is a multi-disciplinary open access archive for the deposit and dissemination of scientific research documents, whether they are published or not. The documents may come from teaching and research institutions in France or abroad, or from public or private research centers.
L'archive ouverte pluridisciplinaire HAL, est destinée au dépôt et à la diffusion de documents scientifiques de niveau recherche, publiés ou non, émanant des établissements d'enseignement et de recherche français ou étrangers, des laboratoires publics ou privés. 


\title{
Geometric synthesis of a hybrid limit cycle for the stabilizing control of a class of nonlinear switched dynamical systems ${ }^{\underline{n}}$
}

\author{
J. Ben Salah ${ }^{\mathrm{a}}$, C. Valentin*,a, H. Jerbi ${ }^{\mathrm{b}}$, C.Z.Xu ${ }^{\mathrm{a}}$ \\ ${ }^{a}$ Université de Lyon, F-69622, Lyon, France; Université Lyon 1, Villeurbanne; LAGEP, UMR 5007, CNRS, CPE, \\ 43 boulevard du 11 Novembre 1918, 69100 villeurbanne, France \\ ${ }^{b}$ University of Sfax, Departement of Math, Faculty of sciences in Sfax, Route de la Soukra km 4 - Sfax - 3038, Tunisie
}

\begin{abstract}
This paper proposes a new constructive method for synthesizing a hybrid limit cycle for the stabilizing control of a class of switched dynamical systems in $\mathbb{R}^{2}$, switching between two discrete modes and without state discontinuity. For each mode, the system is continuous, linear or nonlinear. This method is based on a geometric approach. The first part of this paper demonstrates a necessary and sufficient condition of the existence and stability of a hybrid limit cycle consisting of a sequence of two operating modes in $\mathbb{R}^{2}$ which respects the technological constraints (minimum duration between two successive switchings, boundedness of the real valued state variables). It outlines the established method for reaching this hybrid limit cycle from an initial state, and then stablizing it, taking into account the constraints on the continuous variables. This is then illustrated on a Buck electrical energy converter and a nonlinear switched system in $\mathbb{R}^{2}$. The second part of the paper proposes and demonstrates an extension to $\mathbb{R}^{n}$ for a class of systems, which is then illustrated on a nonlinear switched system in $\mathbb{R}^{3}$.
\end{abstract}

Key words: Switched dynamical system, stability, hybrid limit cycle, nonlinear optimization, stabilizing control.

\section{Introduction}

Switched dynamical systems (SDS) are an important class of hybrid dynamic systems [25] in which the trajectory is continuous or piecewise continuous with controlled or autonomous switches (i.e.with or without a control signal) between different dynamics (or modes). This class of dynamic systems typically

\footnotetext{
This work is a collaboration between LAGEP laboratory of Claude Bernard University, Lyon, France, and Mathematic Departement of Faculty of sciences in Sfax, Tunisia.

* Corresponding author

Email addresses: jben_salah@hotmail.com (J. Ben Salah), valentin@lagep.univ-lyon1.fr (C. Valentin), hjerbi@voila.fr (H. Jerbi), xu@lagep.univ-lyon1.fr (C.Z.Xu)
}

consists of a process with autonomous switching (caused by shocks or using diodes etc) or controlled switching (using transistors, relays, valves, etc.). The controlled switching sequence is the discrete control. The SDS are found in many fields of application: transport, embedded systems, electronics power, aeronautics, chemical engineering, pharmaceutical, etc. It can be seen in these applications that interactions between discrete events and continuous phenomena give rise to complex system behavior that can only be properly controlled if the hybrid phenomena (continuous and discrete features, and interactions between them) are fully taken into consideration $[11,15,16]$.

Significant progress has been made in stability analysis and control synthesis for specific classes of 
hybrid systems (piecewise linear systems, switched systems, etc.). However the field synthesis of control laws with or without constraints remains wide open (robustness, performance, delay, saturation, etc.) $[9,10,11,15,16,17,18,19,21,26]$.

Several important results regarding stability and stabilization of dynamic switched systems are presented in $[4,5,6]$. These provide us with different definitions of stability (total stability, uniform stability, conditional stability, global asymptotic stability, etc.) as well as the relevant conceptual tools (hybrid solution, duration time, common Lyapunov function, multiple Lyapunov functions, convexified system, etc.). In [6], stabilization methods are given in the linear and nonlinear case (uniform stabilization).

If $x_{d}$ is the desired operating point of the SDS and if it is different from the equilibrium points (same or different) of the two discrete modes, if any, the SDS is stable if, and only if, there exists at least one stable hybrid limit cycle around the desired operating point, $x_{d}$, or as close as possible to it [24]. If the technological constraints which impose a minimum duration between two successive switchings are met, this hybrid limit cycle can not be degenerated at a Zeno state. The most commonly used method for stability analysis and systems stabilization, including for switched systems, is based on the Lyapunov theory $[16,7,12,8,6,2,23]$. However, the synthesis of Lyapunov functions is, and has always been, a difficult task especially for nonlinear systems. Some synthesis methods based on quadratic Lyapunov functions have been proposed for Piecewise Linear Systems (PLS), for example, in [14, 20]. In his $\mathrm{PhD}$ thesis [12], Goncalves defines some conditions for the existence and stability of hybrid limit cycles for PLS with known switching surfaces. This means that the control function is also known. Then, over execution time $\tau$, the $\tau$-trajectory ( $\tau$-solution) can be calculated and the existence and the stability of the obtained limit cycle proven.

The approach presented in this paper deals with the synthesis of stabilizing control with unknown switching surfaces. It is an extension of [1].
One widely studied family of switched dynamical systems is that of electrical energy converters, however it remains difficult to synthesize a direct control law for the switching because of the high frequency of their dynamics (or modes). These systems are usually controlled using pulse width modulation (PWM) due to the simplicity with which it can be implemented (output continuous signal compared to a triangular signal). However, this method is unreliable in some cases. For example, in [3] the authors illustrate the instability of multicellular (or multilevel) converters with an odd number of cells under PWM control, and propose a direct and rapid control using a geometric approach which respects an optimal limit cycle with the switching frequency imposed by the characteristics of the semiconductors and which can be synthesized for any number of cells. Another control law is proposed for this converter in [22]. It is based on the switching surfaces calculated using a Lyapunov theory based method which forces the energy of the system to decrease continuously over time.

In the greatest part of this paper, switched dynamic systems (SDS) have a dimension two and switch between two discrete modes without state discontinuity. The main objectives are to define the set of all points around which a hybrid limit cycle can be established (named $E$ in $\mathbb{R}^{2}$ and $\mathcal{E}$ in $\mathbb{R}^{n}$ with $n>2$ ) and to find a hybrid limit cycle which respects the technological constraints (minimum duration between two successive switchings, boundedness of the real valued state variables). It presents a new method, based on the geometric properties of the vector fields [9], and provides an alternative way of addressing the stabilizing control problem. The hybrid limit cycle is determined around a desired operating point, $x_{d}$, or as close as possible to it. This cycle can be reached from an initial state, with a reachability sequence optimized according to time and which meets the physical constraints on the continuous real variables (hypersurfaces threshold) or on the time (minimum duration between two switchings, etc.). A classic method can be used [17]. The threshold hypersurfaces can also be switching surfaces so that the constraints are met. In the 
latter part of this paper, the extension of the main two theorems 3.1 and 3.2 to $\mathbb{R}^{n}$ is presented and demonstrated for a restricted class of systems.

There are other methods to analyse existence and stability of limit cycles in SDS, as Poincare' maps. For example [24] extends the Poincare' Bendixson criterion to hybrid systems with reset functions (i.e. with state discontinuities).

Section 2 sets out some notations and assumptions on geometric properties of vector fields and conditions of existence of a hybrid limit cycle. Section 3 presents and demonstrates the main results of this paper in theorems 3.1 and 3.2. Section 4 formulates the reachability problem in a generic way and section 5 the optimization of the reachability sequence. The algorithm we have developed for the stabilizing control synthesis is given in section 6 and illustrated in section 7 on the linear Buck energy converter and in section 8 on a nonlinear example in $\mathbb{R}^{2}$. Sections 9 and 10 present and demonstrate the extension of the two theorems 3.1 and 3.2 to $\mathbb{R}^{n}$ for a restricted class of systems. Finally, a nonlinear example in $\mathbb{R}^{3}$ is given.

\section{Notations and assumptions}

Assumption 2.1. A continuous trajectory (parameterized arc) in $\mathbb{R}^{2}$ is defined by an enough smooth map $\gamma: I \subseteq \mathbb{R} \longmapsto \mathbb{R}^{2}$.

The Taylor series of $\gamma\left(t+t_{0}\right)$ is:

$$
\gamma\left(t+t_{0}\right)=\gamma\left(t_{0}\right)+\Sigma_{i=1}^{k} \frac{t^{i}}{i !} \gamma^{(i)}\left(t_{0}\right)+t^{k} \varepsilon(t)
$$

where $\varepsilon(t) \rightarrow 0$ when $t \rightarrow 0$ and $\gamma^{(i)}\left(t_{0}\right)$ represents the $i^{t h}$ derivative of $\gamma$ with respect to $t$. We denote $k_{1}$ the smallest strict positive integer such that $\gamma^{\left(k_{1}\right)}\left(t_{0}\right) \neq(0,0)$ and $k_{2}$ the smallest strict positive integer such that $\operatorname{det}\left(\gamma^{\left(k_{1}\right)}\left(t_{0}\right), \gamma^{\left(k_{2}\right)}\left(t_{0}\right)\right) \neq 0$. If $M$ is the point of coordinate $\gamma\left(t+t_{0}\right)$ and $O$ is the point of coordinate $\gamma\left(t_{0}\right)$, it is clear that, for small $t$ :

$$
\overrightarrow{O M}=\left(\frac{t^{k_{1}}}{k_{1} !}+\ldots\right) \overrightarrow{v_{1}}+\frac{t^{k_{2}}}{k_{2} !} \overrightarrow{v_{2}}+t^{k_{2}} \varepsilon(t)
$$

, with $\overrightarrow{v_{1}}=\gamma^{\left(k_{1}\right)}\left(t_{0}\right)\left(\overrightarrow{v_{1}}\right.$ is the tangent vector of the parametrized curve $\gamma$ at $\left.t_{0}\right)$ and $\overrightarrow{v_{2}}=\gamma^{\left(k_{2}\right)}\left(t_{0}\right)$. The shape of the continuous trajectory depends on $k_{2}$ and $k_{1}$. The two possible shapes corresponding to $k_{1}$ odd are represented in figure 1 and the two possible shapes corresponding to $k_{1}$ even are represented in figure 2 (without loss of generality, the vectors $\overrightarrow{v_{1}}$ and $\overrightarrow{v_{2}}$ are represented orthogonally).

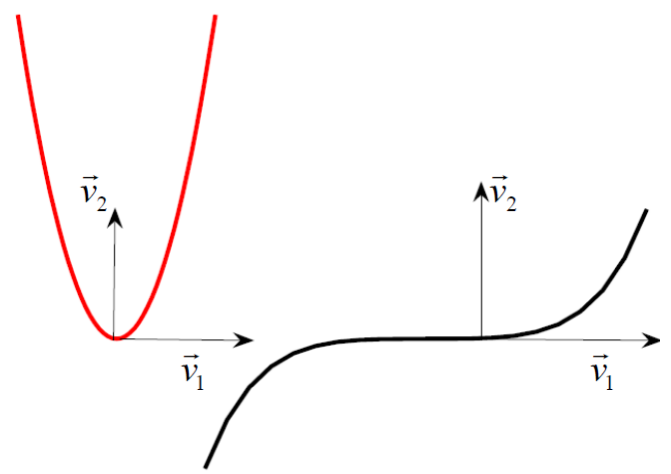

Figure 1: Shapes of the continuous trajectory if $k_{1}$ is odd: on the left, $k_{2}$ is even and on the right, $k_{2}$ is odd.

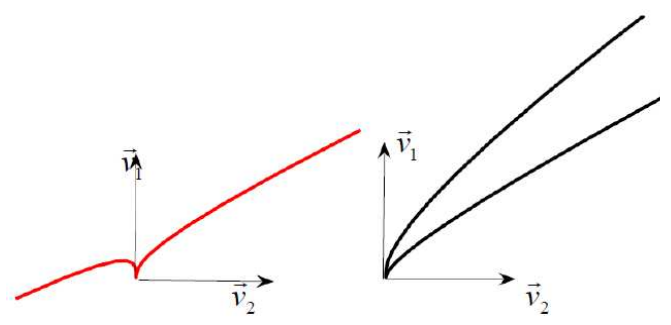

Figure 2: Shapes of the continuous trajectory if $k_{1}$ is even: on the left, $k_{2}$ is odd and on the right, $k_{2}$ is even.

Assumption 2.2. Let $\gamma_{j}: I \subseteq \mathbb{R} \longrightarrow \mathbb{R}^{2}, j \in$ $\{1,2\}$, be two enough smooth maps. Suppose that $\gamma_{1}\left(t_{0}\right)=\gamma_{2}\left(t_{0}\right)$ (the two continuous trajectories intersect at $t_{0}$ at the point $\left.O\right)$ with $\gamma_{1}^{\prime}\left(t_{0}\right) \neq 0$ and $\gamma_{2}^{\prime}\left(t_{0}\right) \neq 0$ and $\operatorname{det}\left(\gamma_{1}^{\prime}\left(t_{0}\right), \gamma_{2}^{\prime}\left(t_{0}\right)\right)=0$ (i.e. $\gamma_{1}^{\prime}\left(t_{0}\right)$ and $\gamma_{2}^{\prime}\left(t_{0}\right)$ are collinear. $\forall j \in\{1,2\}, k_{j 1}=1$ so the two possible shapes of the two continuous trajectories near the point $O$ are represented figure 1). 
The Taylor series of $\gamma_{j}\left(t+t_{0}\right)$ with $j \in\{1,2\}$ are:

$$
\gamma_{j}\left(t+t_{0}\right)=\gamma_{j}\left(t_{0}\right)+\Sigma_{i=1}^{k} \frac{t^{i}}{i !} \gamma_{j}^{(i)}\left(t_{0}\right)+t^{k} \varepsilon_{j}(t)
$$

where $\varepsilon_{j}(t) \rightarrow 0$ when $t \rightarrow 0$.

For SDS which meet the assumption 2.2, the existence of a hybrid limit cycle around the point $O$ depends on the relative position of the two continuous trajectories near this point.

Let us denote $v=\gamma_{1}^{\prime}\left(t_{0}\right), p_{1}$ as the smallest strict positive integer such that $\operatorname{det}\left(\gamma_{1}^{\left(p_{1}\right)}\left(t_{0}\right), v\right) \neq 0$ and $p_{2}$ as the smallest strict positive integer such that $\operatorname{det}\left(\gamma_{2}^{\left(p_{2}\right)}\left(t_{0}\right), v\right) \neq 0$. Without loss of generality, suppose that $p_{1} \leq p_{2}$. Let $M_{j}$ be the point of coordinate $\gamma_{j}\left(t_{j}+t_{0}\right)$. Under these notations, for SDS which meet assumption 2.2, we can write, for small $t_{1}$ and $t_{2}$ :

$$
\begin{aligned}
\overrightarrow{O M}_{1} & =\gamma_{1}\left(t_{1}+t_{0}\right)-\gamma_{1}\left(t_{0}\right) \\
& =t_{1} \gamma_{1}^{\prime}\left(t_{0}\right)+\ldots+\frac{t_{1}^{1}}{p_{1} !} \gamma_{1}^{\left(p_{1}\right)}\left(t_{0}\right)+\ldots \\
\overrightarrow{O M}_{2} & =\gamma_{2}\left(t_{2}+t_{0}\right)-\gamma_{2}\left(t_{0}\right) \\
& =t_{2} \gamma_{2}^{\prime}\left(t_{0}\right)+\ldots+\frac{t_{2}^{2}}{p_{2} !} \gamma_{2}^{\left(p_{2}\right)}\left(t_{0}\right)+\ldots
\end{aligned}
$$

If $t_{2}=\frac{t_{1}\left\langle\gamma_{1}^{\prime}\left(t_{0}\right) \mid v\right\rangle}{\left\langle\gamma_{2}^{\prime}\left(t_{0}\right) \mid v\right\rangle}, M_{1}$ and $M_{2}$ have the same abscissa in the basis $\left(v, v^{\perp}\right)$. Note that $\left\langle\gamma_{2}^{\prime}\left(t_{0}\right) \mid v\right\rangle \neq 0$ because the SDS under consideration meets assumption 2.2 .

Definition 2.1. For SDS which meet the assumption 2.2, $p$ is the smallest positive integer such that:

$$
\begin{aligned}
& \left\langle\gamma_{2}^{\prime}\left(t_{0}\right) \mid \gamma_{1}^{\prime}\left(t_{0}\right)\right\rangle^{p} \operatorname{det}\left(\gamma_{1}^{(p)}\left(t_{0}\right), \gamma_{1}^{\prime}\left(t_{0}\right)\right) \\
& \neq\left\langle\gamma_{1}^{\prime}\left(t_{0}\right) \mid \gamma_{1}^{\prime}\left(t_{0}\right)\right\rangle^{p} \operatorname{det}\left(\gamma_{2}^{(p)}\left(t_{0}\right), \gamma_{1}^{\prime}\left(t_{0}\right)\right) .
\end{aligned}
$$

Note that $\left\langle\gamma_{i}^{(p)}\left(t_{0}\right) \mid v^{\perp}\right\rangle=\operatorname{det}\left(\gamma_{i}^{(p)}\left(t_{0}\right), v\right)$ with $i \in\{1,2\}$ and $v^{\perp}$ a vector orthogonal to $v$.

If $p_{2}>p_{1}$ then $p=p_{1}$ and the difference between $M_{1}$ and $M_{2}$ ordinates in the basis $\left(v, v^{\perp}\right)$, is:

$$
\left\langle\overrightarrow{O M_{1}} \mid v^{\perp}\right\rangle-\left\langle\overrightarrow{O M_{2}} \mid v^{\perp}\right\rangle \cong \frac{t_{1}^{p_{1}}}{p_{1} !} \operatorname{det}\left(\gamma_{1}^{\left(p_{1}\right)}\left(t_{0}\right), v\right)
$$

If $\left(\left(p_{2}=p_{1}\right)\right.$ and $\left.\left(p_{1} \neq p\right)\right)$ then $p>p_{2}$ and the difference between $M_{1}$ and $M_{2}$ ordinates in the basis $\left(v, v^{\perp}\right)$, is:

$$
\begin{aligned}
& \left\langle\overrightarrow{O M_{1}} \mid v^{\perp}\right\rangle-\left\langle\overrightarrow{O M_{2}} \mid v^{\perp}\right\rangle \\
& \cong \frac{t_{1}^{p}}{p !}\left\langle\gamma_{1}^{(p)}\left(t_{0}\right) \mid v^{\perp}\right\rangle-\frac{t_{2}^{p}}{p !}\left\langle\gamma_{2}^{(p)}\left(t_{0}\right) \mid v^{\perp}\right\rangle \\
& \cong \frac{t_{1}^{p}}{p !}\left\langle\gamma_{1}^{(p)}\left(t_{0}\right) \mid v^{\perp}\right\rangle-\frac{t_{1}^{p}}{p !} \frac{\left\langle\gamma_{1}^{\prime}\left(t_{0}\right) \mid v\right\rangle^{p}}{\left\langle\gamma_{2}^{\prime}\left(t_{0}\right) \mid v\right\rangle^{p}}\left\langle\gamma_{2}^{(p)}\left(t_{0}\right) \mid v^{\perp}\right\rangle \\
& \cong \frac{t_{1}^{p}}{p !} \frac{\left(\left\langle\gamma_{2}^{\prime}\left(t_{0}\right) \mid v\right\rangle^{p} \operatorname{det}\left(\gamma_{1}^{(p)}\left(t_{0}\right), v\right)-\left\langle\gamma_{1}^{\prime}\left(t_{0}\right) \mid v\right\rangle^{p} \operatorname{det}\left(\gamma_{2}^{(p)}\left(t_{0}\right), v\right)\right)}{\left\langle\gamma_{2}^{\prime}\left(t_{0}\right) \mid v\right\rangle^{p}}
\end{aligned}
$$

The two parameterized curves are transverse at $t_{0}$ if the sign of $\left(\left\langle\overrightarrow{O M_{1}} \mid v^{\perp}\right\rangle-\left\langle\overrightarrow{O M_{2}} \mid v^{\perp}\right\rangle\right)$ changes when $t_{1}$ changes sign.

Theorem 2.1. For SDS which meet assumption 2.2, the two parameterized curves are transverse if $p$ is odd. Otherwise they are not transverse.

Proof: this theorem comes directly from the equations (1) and (2) because the sign of $\left(\left\langle\overrightarrow{O M_{1}} \mid v^{\perp}\right\rangle-\right.$ $\left.\left\langle\overrightarrow{O M_{2}} \mid v^{\perp}\right\rangle\right)$ depends on the sign of $\frac{t_{1}^{p}}{p !}$. Figure 3 presents two possible respective shapes of $\gamma_{1}$ and $\gamma_{2}$.
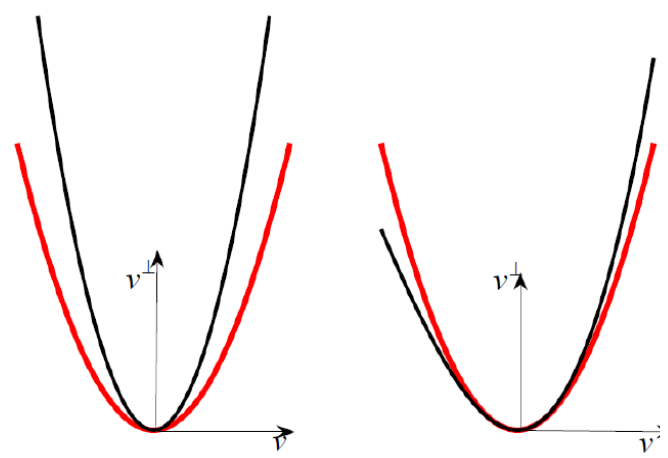

Figure 3: On the left, $p$ is even (curves not transverse) and on the right, $p$ is odd (curves transverse).

Let us consider the time invariant switched dynamic system (SDS) in $\mathbb{R}^{n}$ :

$$
\dot{x}=u f_{1}(x)+(1-u) f_{2}(x)
$$


with the so-called switching function (or discrete control) $u():.\left[\begin{array}{ll}0 & \infty\end{array}\right] \mapsto\{0,1\}$ and $x \in \mathbb{R}^{n}$. It is made of two locations (modes, subsystems) $l_{i}(i \in\{1,2\})$, with respective Lipchitz vector fields $f_{1}(x)$ and $f_{2}(x)$ in $\mathbb{R}^{n}$. There is no state discontinuity at the moment of switching.

The state of the SDS is hybrid (continuous and discrete states): $\left(x, l_{j}\right) \in \mathbb{R}^{n} \times L$ with $L=\left\{l_{j}, j \in\right.$ $\{1,2\}\}$. The (Carathéodory) solution of the differential equation $\dot{x}=f_{i}(x)$ after elapsed time $\delta$ with initial condition $x(0)=x_{0}$ is denoted $\Phi_{i}\left(x_{0}, \delta\right)$, $i \in\{1,2\}$. In section 4 and 5 , we also use the following notation: $\Phi_{i}^{\delta}\left(x_{0}\right), i \in\{1,2\}$.

If both modes $l_{1}$ and $l_{2}$ have an equilibrium point $x_{e 1}$ and $x_{e 2}$ (same or different), and are globally asymptotically stable, then, for all $x_{0} \in \mathbb{R}^{n}$ and $i \in\{1,2\}, \lim _{\delta \rightarrow \infty} \Phi_{i}\left(x_{0}, \delta\right)=x_{e i}$. If $x_{d}=x_{e 1}$ or $x_{d}=x_{e 2}$, then, the desired operating point $x_{d}$ can be reached asymptotically.

Definition 2.2. Let us consider $x_{c 1}$ and $x_{c 2}$ two points in $\mathbb{R}^{n}$, with $x_{c 1} \neq x_{c 2} . C C\left(x_{c 1}, x_{c 2}\right)$ is the hybrid limit cycle of the $S D S \dot{x}=f_{i}(x), i \in\{1,2\}$, between the switching points $x_{c 1}$ and $x_{c 2}$, if and only if $\left(\delta_{c 1}, \delta_{c 2}\right) \in \mathbb{R}_{+}^{2}$ exists such that: $x_{c 1}=\Phi_{1}\left(x_{c 2}, \delta_{c 1}\right)$ and $x_{c 2}=\Phi_{2}\left(x_{c 1}, \delta_{c 2}\right)$. Then

$$
\begin{gathered}
C C\left(x_{c 1}, x_{c 2}\right)=\left\{\Phi_{1}\left(x_{c 2}, \delta\right) / 0 \leq \delta \leq \delta_{c 1}\right\} \cup \\
\left\{\Phi_{2}\left(x_{c 1}, \delta\right) / 0 \leq \delta \leq \delta_{c 2}\right\} .
\end{gathered}
$$

If $x_{d} \neq x_{e 1}$ and $x_{d} \neq x_{e 2}$ or if at least one of the subsystems is unstable, stabilizing a SDS whilst respecting the technological constraints involves determining a stable hybrid limit cycle as close as possible to $x_{d}$.

Definition 2.3. In the plane, a loop $L_{\gamma}$ is defined by a continuous map in the interval $[a, b], \gamma:[a, b] \rightarrow$ $\mathbb{R}^{2}, t \mapsto \gamma(t)$ such that $\gamma(a)=\gamma(b)$. The interior of the loop $L_{\gamma}=\gamma([a, b])$ is the union of open sets bounded by this loop. This is denoted as $\operatorname{Int}\left(L_{\gamma}\right)$.

An example in which the interior of the loop is formed from a single open bounded set is given in figure 4 .

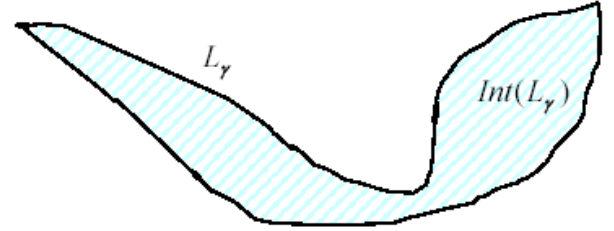

Figure 4: Example of the interior of a loop $L_{\gamma}$ in the plane.

A hybrid limit cycle in $\mathbb{R}^{2}, C C\left(x_{c 1}, x_{c 2}\right)$, with at least one point $x \in C C\left(x_{c 1}, x_{c 2}\right)$ such that $f_{1}(x) \neq$ $-f_{2}(x)$, is a loop. Since $x_{c 1} \neq x_{c 2}$, such a hybrid limit cycle is not degenerated at a single point. If $\forall x \in$ $C C\left(x_{c 1}, x_{c 2}\right), f_{1}(x)=-f_{2}(x)$, the hybrid limit cycle is made up of two opposite trajectories and reduced to a curve, the trajectory making a roundtrip between the two switching points, and $\delta_{c 1}=\delta_{c 2}$. An example of a hybrid limit cycle in $\mathbb{R}^{2}$ with at least one point $x \in C C\left(x_{c 1}, x_{c 2}\right)$ such that $f_{1}(x) \neq-f_{2}(x)$ is given in figure 5 .

Note that a hybrid limit cycle is a closed trajectory made up of two dynamics unlike a periodic orbit in the real valued state space that is generated by a single continuous dynamic (one time invariant state space representation $\dot{x}=f(x)$ with $\left.x \in \mathbb{R}^{n}\right)$.

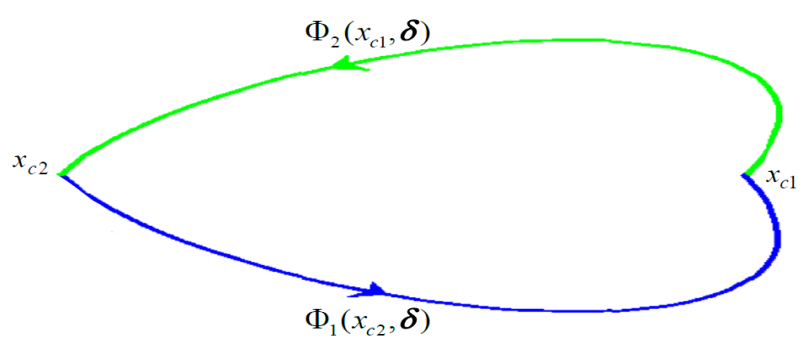

Figure 5: Example of hybrid limit cycle for a SDS in $\mathbb{R}^{2}$.

\section{A necessary and sufficient condition of ex- istence and stability of a hybrid limit cycle}

Notation: Let us denote as $d^{p-1} f_{i}(x)=$ $\left.\frac{d^{p} \Phi_{i}(x, t)}{d t^{p}}\right|_{t=0}$ with $p \geq 1$.

$v=f_{1}(x) \neq 0, p_{1}$ is the smallest integer such that $p_{1}>1$ and $\operatorname{det}\left(d^{p_{1}-1} f_{1}(x), v\right) \neq 0$ and $p_{2}$ the smallest 
integer such that $p_{2}>1$ and $\operatorname{det}\left(d^{p_{2}-1} f_{2}(x), v\right) \neq 0$. From definition $(2.1), p(x)$ is the smallest positive integer such that:

$$
\begin{aligned}
& \left\langle f_{2}(x) \mid v\right\rangle^{p} \operatorname{det}\left(d^{p-1} f_{1}(x), v\right) \\
& \neq\left\langle f_{1}(x) \mid v\right\rangle^{p} \operatorname{det}\left(d^{p-1} f_{2}(x), v\right) .
\end{aligned}
$$

Definition 3.1. $E=\left\{z \in \mathbb{R}^{2} / \operatorname{det}\left(f_{1}(z), f_{2}(z)\right)=\right.$ $0,\left\langle f_{1}(z) \mid f_{2}(z)\right\rangle<0$ and $p(z)$ is even $\}$ is the set of points in $\mathbb{R}^{2}$ with collinear and opposite vector fields $f_{i}(x), i \in\{1,2\}$, and with non transverse trajectories.

Let us recall that $\operatorname{Int}\left(C C\left(x_{c 1}, x_{c 2}\right)\right) \cup C C\left(x_{c 1}, x_{c 2}\right)$ is denoted as $\overline{\operatorname{Int}\left(C C\left(x_{c 1}, x_{c 2}\right)\right)}$. $\overline{\operatorname{Int}\left(C C\left(x_{c 1}, x_{c 2}\right)\right)}$ is the closure of $\operatorname{Int}\left(C C\left(x_{c 1}, x_{c 2}\right)\right)$.

Theorem 3.1. Let us consider a SDS (3) with $x \in \mathbb{R}^{2}$. Let $C C\left(x_{c 1}, x_{c 2}\right)$ in $\mathbb{R}^{2}$ be a hybrid limit cycle with $x_{c 1} \neq x_{c 2}$ with at least one point $x \in C C\left(x_{c 1}, x_{c 2}\right)$ such that $f_{1}(x) \neq-f_{2}(x)$. If the closure $\overline{\operatorname{Int}\left(C C\left(x_{c 1}, x_{c 2}\right)\right)}$ does not include any of the equilibrium points of the two dynamics $f_{1}$ and $f_{2}$, then, there exists a non-empty set of points $z \in \mathbb{R}^{2}$, such that $z \in E \bigcap \overline{\operatorname{Int}\left(C C\left(x_{c 1}, x_{c 2}\right)\right)}$ i.e $E \bigcap \overline{\operatorname{Int}\left(C C\left(x_{c 1}, x_{c 2}\right)\right)} \neq \phi$.

Proof: Let us consider the hybrid cycle $C C\left(x_{c 1}, x_{c 2}\right)$. Some part of the trajectory, defined by $A=\left\{\Phi_{2}\left(x_{c 1}, t\right)\right.$ with $\left.0 \leq t \leq \delta_{c 2}\right\}$ is fixed. For any points $x_{c}$ of $A$, the trajectories with dynamic $f_{1}(x)$, starting from $x_{c}$ may have three shapes because there is at least one point $x \in C C\left(x_{c 1}, x_{c 2}\right)$ such that $f_{1}(x) \neq-f_{2}(x)$. First, consider the trajectories that renter inside $\overline{\operatorname{Int}\left(C C\left(x_{c 1}, x_{c 2}\right)\right)}$. Necessarily, $\delta_{c}>0$ exists such that $x_{\tilde{c}}=\Phi_{1}\left(x_{c}, \delta_{c}\right) \in A$ because $\overline{\operatorname{Int}\left(C C\left(x_{c 1}, x_{c 2}\right)\right)}$ does not include an equilibrium point of the dynamic $f_{1}$. Since two trajectories from the same vector field do not intersect, it follows that $C C\left(x_{c}, x_{\tilde{c}}\right) \subset \overline{\operatorname{Int}\left(C C\left(x_{c 1}, x_{c 2}\right)\right)}$. Secondly, let us consider the trajectories that leave $\overline{\operatorname{Int}\left(C C\left(x_{c 1}, x_{c 2}\right)\right)}$. In this case, $\delta_{c}>0$ exists such that $x_{\tilde{c}}=\Phi_{1}\left(x_{c},-\delta_{c}\right) \in A$, then $C C\left(x_{c}, x_{\tilde{c}}\right) \subset$ $\overline{\operatorname{Int}\left(C C\left(x_{c 1}, x_{c 2}\right)\right)}$. In the third case, the trajectory starting from $x_{c}$ with dynamic $f_{1}(x)$ is tangent to $A$ at $x_{c}$. Figure 6 represents cases 1 and 3. Case 2 can be easily deduced.

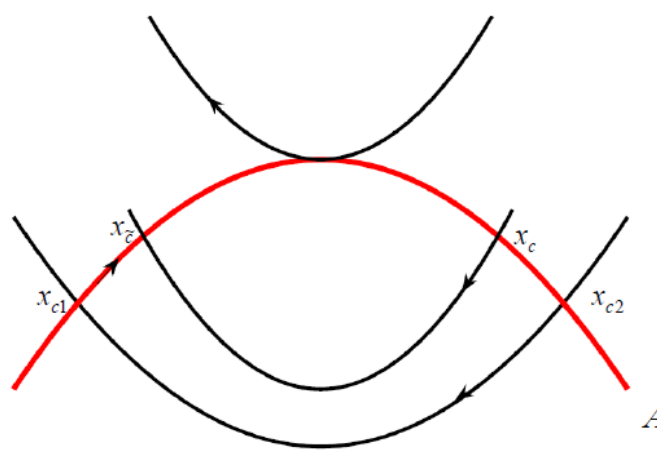

Figure 6: Geometric motivation of thorem 3.1.

In the following, it is proved that a point $x_{c}$ necessarily exists in $A$ and that this point verifies the third condition and belongs to $E$.

For any point $x_{c}$ in $A$ a hybrid cycle $C C\left(x_{c}, x_{\tilde{c}}\right)$ can be constructed. Since two trajectories from the same vector field do not intersect, then for any two points $x_{c}$ and $x_{c^{\prime}}$ in the set $A$, we have:

$$
\overline{\operatorname{Int}\left(C C\left(x_{c}, x_{\tilde{c}}\right)\right)} \subset \overline{\operatorname{Int}\left(C C\left(x_{c^{\prime}}, x_{\tilde{c}^{\prime}}\right)\right)}
$$

or

$$
\overline{\operatorname{Int}\left(C C\left(x_{c^{\prime}}, x_{\tilde{c}^{\prime}}\right)\right)} \subset \overline{\operatorname{Int}\left(C C\left(x_{c}, x_{\tilde{c}}\right)\right)}
$$

This creates a non-empty closed set, $\mathcal{A}$, of hybrid limit cycles with switching points belonging to the fixed path $A$ :

$$
\mathcal{A}=\left\{\overline{\operatorname{Int}\left(C C\left(x_{c}, x_{\tilde{c}}\right)\right)} / x_{c} \in A\right\}
$$

Note that $C C\left(x_{c}, x_{\tilde{c}}\right) \subseteq \overline{\operatorname{Int}\left(C C\left(x_{c 1}, x_{c 2}\right)\right)}$. The elements of $\mathcal{A}$ are nested one inside each other and are ordered by set inclusion. According to Zorn's lemma [27] (Any inductive, ordered and non-empty set admits at least a minimum element), this set accepts a minimum that cannot be a cycle, because within a cycle, we can always find another cycle (continuity of the two vector fields), but a point, z. So, the intersection between the fixed trajectory, $A$, and the trajectory with dynamic $f_{1}($.$) passing through z$, is reduced to this point $z$. As this trajectory is tangent to $A$ at $z, \operatorname{det}\left(f_{1}(z), f_{2}(z)\right)=0$. Moreover, $\left\langle f_{1}(z) \mid f_{2}(z)\right\rangle>0$ would mean that there is no cycle around this point, 
which contradicts the fact that $z$ is the minimum element, thus $\left\langle f_{1}(z) \mid f_{2}(z)\right\rangle<0$. As $z$ is the minimum element of a set of hybrid limit cycles, the trajectory with dynamic $f_{1}($.$) passing through z$ is not transverse with $A$, then $z \in E$. This completes the proof.

Theorem 3.2. Let us consider the SDS (3) with $x \in \mathbb{R}^{2}$. For each point $z \in E \neq \phi$, there exists a hybrid limit cycle $C C\left(x_{c 1}, x_{c 2}\right)$ such that $z \in$ $\overline{\operatorname{Int}\left(C C\left(x_{c 1}, x_{c 2}\right)\right)}$.

Proof: $\forall z \in E, f_{1}(z)=\alpha f_{2}(z)$ with $\alpha<0$ and the trajectories issued from $z$ with dynamic $f_{1}$ and $f_{2}$ are not transverse in $z$. Figures 7.a and 7.b represent two possible trajectory shapes in the neighbourhood of $z$. As the trajectories from $z$ with dynamic $f_{1}$ and $f_{2}$ are not transverse in $z$, it follows that two trajectories from these two vector fields in the neighbourhood of $z$ create a hybrid limit cycle. Figures 8.a and 8.b represent two possible shapes in the state space. Therefore, a hybrid limit cycle can always be designed around the point $z$, where it exists. This completes the proof.

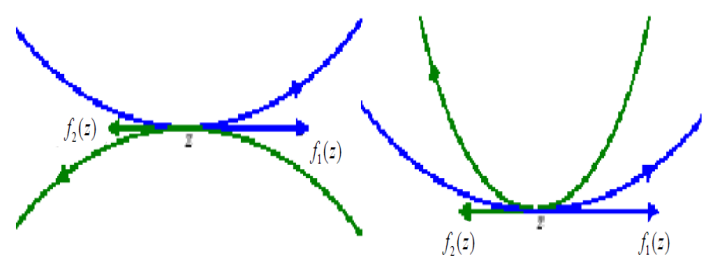

(a)

(b)

Figure 7: Sections of trajectories passing through $z \in E$ in $I^{2}$.

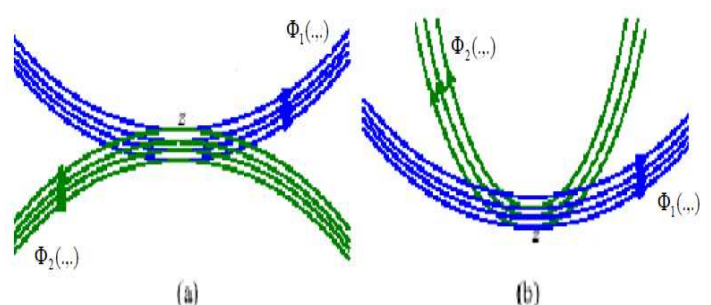

Figure 8: Geometric motivation of theorem 3.2.

Note that there exists an infinity of hybrid limit cycles around each point of the non-empty set $E$. Then, if $x_{d} \in E$, there exists a hybrid limit cycle, between two different switching points, where the SDS (3) can be stabilized. If $x_{d}$ does not belong to $E$, it is necessary to find the nearest point belonging to the non-empty set $E$, around which the SDS can be stabilized. Note that the physical constraints imposed by the system (minimum duration time between two switchings, states thresholds, etc.) must also be fulfilled.

Note that $C C\left(x_{d}, x_{d}\right)$ is a stable cycle degenerated at the point $x_{d}$. It has a physical meaning only if $x_{d}=x_{e 1}$ or $x_{d}=x_{e 2}$. Otherwise, the SDS is Zeno, i.e there are an infinite number of switchings at a finite time and the period of the cycle becomes null.

\section{Reachability domain}

Let us now recall the classic method for reaching the hybrid limit cycle determined in the previous section, from an initial state, and stabilizing it, with respect to the constraints on the continuous variables. Let us formulate this reachability problem in a generic way.

A trajectory (or solution) of a SDS from a hybrid initial state $\left(x_{0}, l_{i}\right)$ with $i \in\{1,2\}$ to a hybrid final state $\left(x_{c}, l_{k}\right) \in C C\left(x_{c 1}, x_{c 2}\right)$ is defined as follows: $x_{0} \rightarrow x_{1}=\Phi_{i}\left(x_{0}, \delta_{s_{1}}\right) \rightarrow x_{2}=\Phi_{j}\left(x_{1}, \delta_{s_{2}}\right) \rightarrow \ldots \rightarrow$ $x_{m}=\Phi_{i}\left(x_{m-1}, \delta_{s_{m}}\right) \rightarrow \ldots \rightarrow x c=\Phi_{k}\left(x_{c-1}, \delta_{s_{c}}\right)$ with $(i, j, k) \in\{1,2\}^{3}$ and $\forall m \in\{1, \ldots, c\}$, $\delta_{s_{m}} \in \mathbb{R}_{+}$with $\delta_{s_{m}}$ the duration time in the active subsystem (or mode) before the $m^{\text {th }}$ switching in the sequence $S$. This continuous trajectory is made up of a succession of continuous trajectories with different dynamics. The concatenation of dynamics (discrete states) defines the switching sequence $S=\left(l_{s_{1}}, l_{s_{2}}, \ldots, l_{s_{c}}\right)$ with $s_{k} \in\{1,2\}$ and $1 \leq k \leq c$. The switching points are $\left(x_{1}, x_{2}, \ldots, x_{c}\right)$. The SDS vector field may be discontinuous at the moment of switching, but there is no state discontinuity. The total duration of time the SDS takes to reach the hybrid limit cycle $C C\left(x_{c 1}, x_{c 2}\right)$ from the initial point is therefore: $t_{c}=\delta_{s_{1}}+\delta_{s_{2}}+\ldots+\delta_{s_{c}}$.

The global constraints of the system define the global 
operating domain which is denoted as $\Omega$. It can be represented using the $g$ following linear inequalities: $\forall k \in\{1, \ldots, g\}, C_{k} x+d_{k} \leq 0$ with $C_{k}$ of dimension $n$ and $d_{k}$ is a constant.

Definition 4.1. Let us consider a hybrid limit cycle $C C\left(x_{c 1}, x_{c 2}\right)$. We define:

$A_{1}=\left\{\Phi_{1}(z,-\delta) \in \Omega, \forall z \in C C\left(x_{c 1}, x_{c 2}\right)\right.$ and $\left.\delta \geq 0\right\}$

$B_{1}=\left\{\Phi_{2}(z,-\delta) \in \Omega, \forall z \in C C\left(x_{c 1}, x_{c 2}\right)\right.$ and $\left.\delta \geq 0\right\}$ :

$A_{i+1}=\left\{\Phi_{1}(z,-\delta) \in \Omega, \forall z \in B_{i}\right.$ and $\left.\delta \geq 0\right\}$

$B_{i+1}=\left\{\Phi_{2}(z,-\delta) \in \Omega, \forall z \in A_{i}\right.$ and $\left.\delta \geq 0\right\}$

$\vdots$

These are the sets of trajectories that define all backward inferences from the hybrid limit cycle $C C\left(x_{c 1}, x_{c 2}\right)$ following the two vector field flows. It should be noted that these $A_{i}$ and $B_{i}$ do not necessarily define a partition of the state space.

A necessary and sufficient condition for SDS reachability by switching between two vector fields in $\mathbb{R}^{n}$ is given in the following theorem 4.1:

Theorem 4.1. Let us consider that $D=$ $\left(\bigcup_{i>1} A_{i}\right) \bigcup\left(\bigcup_{i>1} B_{i}\right) \subseteq \mathbb{R}^{n}$ is the global reachability domain of the SDS. If $x_{0} \in D$, there exists at least a sequence with a finite number of switchings, which leads the state of the system from the point $x_{0}$ to the hybrid limit cycle $C C\left(x_{c 1}, x_{c 2}\right)$.

Proof: If we consider that $x_{0} \in D$, then there exists an integer $J \geq 1$ such that $x_{0} \in A_{J}$ (without loss of generality because $\left.A_{J} \subset B_{J+1}\right)$. Then, from definition 4.1 and because $D=\left(\bigcup_{i>1} A_{i}\right) \bigcup\left(\bigcup_{i>1} B_{i}\right)$, $\exists \delta_{s_{1}} \in \mathbb{R}_{+}$and $\exists z_{J-1} \in B_{J-1}$ such that $x_{0}=\Phi_{1}^{-\delta_{s_{1}}}\left(z_{J-1}\right)$ and $\exists \delta_{s_{2}} \in \mathbb{R}_{+}$and $\exists z_{J-2} \in A_{J-2}$ such that $z_{J-1}=\Phi_{2}^{-\delta_{s_{2}}}\left(z_{J-2}\right), \cdots$

By inference, $\exists x_{c} \in C C\left(x_{c 1}, x_{c 2}\right)$ and $\exists \delta_{s_{c}} \in \mathbb{R}_{+}$ such that: $x_{0}=\Phi_{1}^{-\delta_{s_{1}}} \circ \Phi_{2}^{-\delta_{s_{2}}} \circ \Phi_{1}^{-\delta_{s_{3}}} \circ \cdots \circ \Phi_{k}^{-\delta_{s_{c}}}\left(x_{c}\right)$

Therefore,

$x_{c}=\Phi_{k}^{\delta_{s_{c}}} \circ \cdots \circ \Phi_{1}^{\delta_{s_{3}}} \circ \Phi_{2}^{\delta_{s_{2}}} \circ \Phi_{1}^{\delta_{s_{1}}}\left(x_{0}\right)$
After the selection of a hybrid limit cycle $C C\left(x_{c 1}, x_{c 2}\right)$, all the possible trajectories obtained by backward inferences from this cycle and meeting the global operating domain $\Omega$ can be determined, according to definition 4.1. If $D$ covers the totality of $\Omega$, then, the hybrid limit cycle can be reached by switching from all point $x_{0}$ in $\Omega$. Otherwise, the initial point $x_{0}$ must be in $D \subset \Omega$, so that there is at least one possible switching sequence that leads the SDS from this point $x_{0}$ to the hybrid limit cycle $C C\left(x_{c 1}, x_{c 2}\right)$. This analysis involves calculating all the state space regions from which the final hybrid limit cycle can be reached.

If one is interested only in finding a hybrid switching sequence that drives the system from the initial state $x_{0}$ to the final hybrid limit cycle $C C\left(x_{c 1}, x_{c 2}\right)$, the analysis is completed as soon as the initial state is included into one of these regions. This analysis is done backwards in the continuous state space. For more details see $[17,18]$.

\section{Reachability sequence optimization}

Let $S$ be a switching sequence obtained by switching between the two vector fields $f_{i}(x)$ with $i \in\{1,2\}$ that defines a trajectory of the SDS from the initial hybrid state $\left(x_{0}, l_{i}\right)$ to a final hybrid state $\left(x_{c}, l_{k}\right) \in C C\left(x_{c 1}, x_{c 2}\right) . \quad c$ is the length of $S$ i.e. the number of switchings in the sequence $S$. $f_{s_{k}}(x)$ with $1 \leq k \leq c$ and $s_{k} \in\{1,2\}$, is the vector field active in the $k^{t h}$ mode $l_{s_{k}}$ of $S$ during a time $\delta_{s_{k}}$ called duration time. $x_{0}$ and $x_{c}$ are respectively the initial and the final continuous states of SDS trajectory. $t_{c}$ is the total duration time for the system to reach $x_{c}$ from $x_{0}$ with the switching sequence, $S$. The optimization problem involves minimizing $t_{c}$, which depends on the duration times $\delta_{s_{k}}$.

For all $t$ such that $0 \leq t \leq t_{c}$, the continuous state, $x$, must belong to the global reachability domain of the SDS, $D$. In this problem, we must also ensure that the trajectory leads to the hybrid limit cycle $C C\left(x_{c 1}, x_{c 2}\right)$. 
So, the problem can be written as the following constrained minimization problem,

$$
t_{c}=\min _{S}\left(\sum_{k=1}^{c} \delta_{s_{k}}\right)
$$

such that,

- $\forall x \in D, \exists s_{k} \in S / \forall \delta \in\left[\begin{array}{ll}0 & \delta_{s_{k}}\end{array}\right], \Phi_{s_{k}}^{\delta}(x) \in D$,

- $x_{c}=\Phi_{s_{c}}^{\delta_{s_{c}}} \circ \cdots \circ \Phi_{s_{2}}^{\delta_{s_{2}}} \circ \Phi_{s_{1}}^{\delta_{s_{1}}}\left(x_{0}\right) \in C C\left(x_{c 1}, x_{c 2}\right)$.

Two methods to solve this problem are given in [17, 18]. In the litterature, the reachability analysis has been realized by many other methods as explained for instance in [13].

\section{Stabilizing control synthesis algorithm}

The algorithm for the synthesis of a stabilizing control for the SDS (3) has six steps:

1. Write the SDS model in the form: $\dot{x}=f_{i}(x)$ with $i \in\{1,2\}$.

2. Determine the set $E$ given in definition 3.1:

- If $E \neq \phi$, then at least a hybrid limit cycle exists and the SDS can be stabilized around all points of $E$. Go to step 3.

- If $E=\phi$, no switching sequence exists to stabilize the SDS around any point of the continuous state space. The SDS cannot be stabilized.

3. After choosing the desired operating point, $x_{d}$, determine $x_{d E}$, the point of $E$ around which a hybrid limit cycle $C C\left(x_{c 1}, x_{c 2}\right)$ can be designed:

- If $x_{d} \in E$, then $x_{d E}=x_{d}$.

- Otherwise, $x_{d E} \in E$ is such that $d\left(x_{d E}, x_{d}\right)=\min _{z \in E} d\left(z, x_{d}\right)$.

4. Determine a hybrid limit cycle $C C\left(x_{c 1}, x_{c 2}\right)$ around $x_{d E}$ that meets the desired specifications. By definition, a hybrid limit cycle whose interior includes $x_{d E}$ always exists but not necessarily one that meets the technological constraints $\left(\delta_{c 1}>\delta_{\min }\right.$ and $\delta_{c 2}>\delta_{\min }, \delta_{\min }$ is the minimum duration time between two successive switchings (dwell time), the state variables are bounded by specific values, the cycle duration time, $\delta_{c 1}+\delta_{c 2}$, is bounded, etc.). This hybrid limit cycle is not unique.

- If there is such a hybrid limit cycle, go to Step 5.

- Otherwise, remove a constraint or choose another point $x_{d E}$.

5. Determine backward (according to definition 4.1), all regions from which the hybrid limit cycle $C C\left(x_{c 1}, x_{c 2}\right)$ can be reached whilst respecting the global operating constraints. If one is interested only in finding a hybrid switching sequence that drives the system from the initial state $x_{0}$ to the final hybrid limit cycle $C C\left(x_{c 1}, x_{c 2}\right)$, the algorithm is completed as soon as the initial state $x_{0}$ is included into one of these regions. If possible, all the regions included in $\Omega$ can be calculated and all the possible switching sequences that drive the system from the initial hybrid state to the desired hybrid limit cycle are made known.

6. Calculate the switching instants of a reachability sequence found in the previous step to obtain the shortest total reaching time, $t_{c}$.

\section{Application to the Buck energy converter}

In this section, the control synthesis algorithm presented in the previous section is applied to the Buck converter control. The usual Buck circuit is given in Figure 9. T and D represent the system switches (electrical semi-conductors). D is a self-switching diode and $\mathrm{T}$ is a controlled transistor (or thyristor). This system operates in continuous mode. The case with both switches open is not considered (no discontinuity of the current through the inductance). The case with both switches closed is not considered either, short-circuiting the voltage source. Thus, only two SDS modes of operation are used: mode $l_{1}$ with $\mathrm{T}$ closed and D blocked and mode $l_{2}$ with $\mathrm{T}$ open and $\mathrm{D}$ closed. If $\mathrm{T}$ is closed, the load receives energy from the source. If $\mathrm{T}$ is opened, the energy is dissipated 
by the load.

The goal is to define a hybrid limit cycle for this system, as near as possible to the desired operating point $x_{d}$, as well as to define a trajectory for reaching this cycle that meets the desired specifications (operating and technological constraints). The synthesized control sequence defines the switching signal.

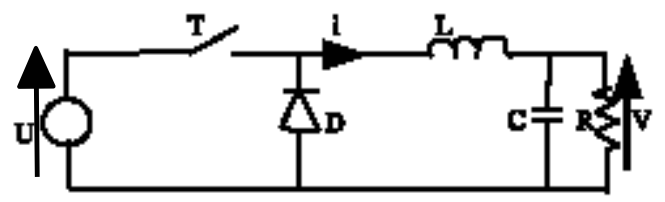

Figure 9: The Buck energy converter.

Now let us apply the control synthesis algorithm presented in the previous section to the following Buck circuit: $U=20 \mathrm{~V}, L=20 \mathrm{mH}, C=47 \mu \mathrm{F}$, $R=22 \Omega$.

The model of the Buck converter is $\dot{x}=f_{i}(x)$ with $i \in\{1,2\}$ and $x=\left[\begin{array}{ll}V & i\end{array}\right]^{T}=\left[\begin{array}{ll}x_{1} & x_{2}\end{array}\right]^{T}$

$$
\left\{\begin{array}{l}
f_{1}(x)=A x+B=\left[\begin{array}{cc}
\frac{-1}{R C} & \frac{1}{C} \\
\frac{-1}{L} & 0
\end{array}\right] x+\left[\begin{array}{c}
0 \\
\frac{U}{L}
\end{array}\right] \\
f_{2}(x)=A x=\left[\begin{array}{cc}
\frac{-1}{R C} & \frac{1}{C} \\
\frac{-1}{L} & 0
\end{array}\right] x
\end{array}\right.
$$

This SDS is a Piecewise Linear System (PLS).

Let us now calculate the set $E=\{z \in$ $\mathbb{R}^{2} / \operatorname{det}\left(f_{1}(z), f_{2}(z)\right)=0$ and $\left\langle f_{1}(z) \mid f_{2}(z)\right\rangle<0$ and $p(z)$ is even $\}$ :

$$
\begin{aligned}
& \left\{\begin{array}{l}
x_{1}=R x_{2} \\
\left(-\frac{x_{1}}{R C}+\frac{x_{2}}{C}\right)^{2}+\frac{x_{1}}{L^{2}}\left(x_{1}-U\right)<0 \\
x_{1} \neq U \text { such that } p_{1}=p_{2}=p=2
\end{array}\right. \\
& \Longleftrightarrow x_{1}\left(x_{1}-U\right)<0 \Longleftrightarrow 0<x_{1}<U
\end{aligned}
$$

Thus, $E=\left\{z \in \mathbb{R}^{2} / z_{1}=R z_{2}\right.$ and $\left.0<z_{1}<U\right\}$ is part of a straight line with slope $\frac{1}{R}$ and end points $(0,0)$ and $\left(U, \frac{U}{R}\right)$ (the straight line that connects the two stable equilibrium points of the two Buck converter modes of operation, $x_{e 1}$ and $x_{e 2}$ ). $E$ is the set of all points around which a hybrid limit cycle may be synthesized for this system.

Figure 10 gives the possible trajectories for the two modes of operation of the Buck converter with vector fields $f_{1}(z)$ (blue curves) and $f_{2}(z)$ (red curves) as well as the set $E$ (part of a straight line in black color). One can intuitively verify the properties of the points which belong to $E$. If $x_{d}$

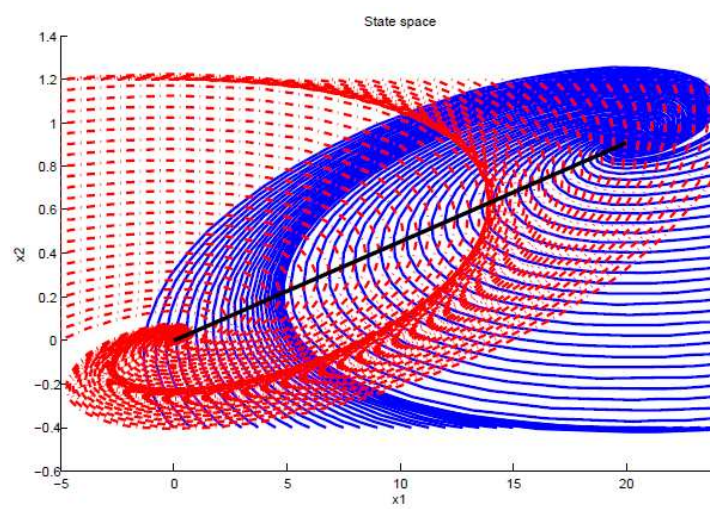

Figure 10: Buck converter trajectories and set E.

does not belong to the set $E, x_{d E}$ is chosen so that $d\left(x_{d E}, x_{d}\right)=\min _{z \in E} d\left(z, x_{d}\right)$. If $C C\left(x_{c 1}, x_{c 2}\right)$ is a hybrid limit cycle, according to the equations (4):

$$
\begin{aligned}
x_{c 1} & =\Phi_{1}\left(x_{c 2}, \delta_{c 1}\right) \\
& =e^{A \delta_{c 1}} x_{c 2}+\int_{0}^{\delta_{c 1}} e^{A\left(\delta_{c 1}-t\right)} B d t \\
x_{c 2} & =\Phi_{2}\left(x_{c 1}, \delta_{c 2}\right)=e^{A \delta_{c 2}} x_{c 1}
\end{aligned}
$$

with $\delta_{c 1}$ (respectively $\delta_{c 2}$ ) the duration time in mode $l_{1}$ (respectively $l_{2}$ ) of the hybrid limit cycle. The switching points are:

$$
\left\{\begin{array}{l}
x_{c 1}=\left(I-e^{A\left(\delta_{c 1}+\delta_{c 2}\right)}\right)^{-1} A^{-1}\left(e^{A \delta_{c 1}}-I\right) B \\
x_{c 2}=e^{A \delta_{c 2}} x_{c 1}
\end{array}\right.
$$

If $x_{c 1}$ is chosen near $x_{d E}$, the choice of $\delta_{c 1}$ and $\delta_{c 2}$ 
determines a hybrid limit cycle whose interior includes $x_{d E} . x_{c 2}$ is given in equations (5). According to theorem 3.2 such a cycle always exists.

The global reachability domain $D$ is obtained according to the backward inference method presented in definition 4.1 and by the property of theorem 4.1, given that $\Omega=\left\{x \in \mathbb{R}^{2} / 0 \leq x_{1} \leq x_{1 \max }\right.$ and $\left.0 \leq x_{2} \leq x_{2 \max }\right\}$. Thus, the system is controllable, and hybrid sequences exist which drive the system from an initial state into the global reachability domain $D$ to the hybrid limit cycle $C C\left(x_{c 1}, x_{c 2}\right)$.

The synthesis and simulation results are given in figure 11. The desired operating point is $x_{d}=$ $\left[\begin{array}{llll}15 & V & 0.7 & A\end{array}\right]$. The choice of $\delta_{c 1}=0.24 \mathrm{~ms}$ and $\delta_{c 2}=0.33 \delta_{c 1}$ defines a cycle around $x_{d}$ with the switching points $x_{c 1}=\left[\begin{array}{lllll}15.007 & V & 0.711 & A\end{array}\right]$ and

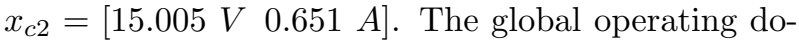
main is defined by: $x_{1 \max }=25 \mathrm{~V}, x_{2 \max }=1.2 \mathrm{~A}$ and $x_{1 \text { min }}=0 \mathrm{~V}$ and $x_{2 m i n}=0 A$. Then, the possible and admissible control sequences from the initial point $x_{0}=\left[\begin{array}{ll}0 & 0\end{array}\right]^{T}$ and final point $x_{f}=$ $\left[\begin{array}{ll}14.968 & 0.681\end{array}\right]^{T}$ which belongs to $\Phi_{1}\left(x_{c 2}, \delta\right)$ with $0<\delta<\delta_{c 1}$ are $\left(l_{1}, l_{2}\right)^{k}, l_{2}\left(l_{1}, l_{2}\right)^{k},\left(l_{1}, l_{2}\right)^{k} l_{1}$ or $l_{2}\left(l_{1}, l_{2}\right)^{k} l_{1}$ with $k \in \mathbb{N}$. The switching sequence with minimum time duration is $\left(l_{1}, l_{2}\right)$ and each mode duration time is $\delta_{s_{1}}=1.407 \mathrm{~ms}$ and $\delta_{s_{2}}=0.565 \mathrm{~ms}$. Thus, $t c=1,972 \mathrm{~ms}$.

\section{Nonlinear example in $\mathbb{R}^{2}$}

Here, the algorithm presented in section 6 is applied to the stabilizing control of a nonlinear SDS, $\dot{x}=f_{i}(x), x=\left[\begin{array}{ll}x_{1} & x_{2}\end{array}\right]^{T}$ with the following vector fields:

$$
\left\{\begin{array}{l}
f_{1}(x)=\left(\begin{array}{l}
-2 x_{1}-x_{1}^{3}-5 x_{2}-x_{2}^{3} \\
6 x_{1}+x_{1}^{3}-3 x_{2}-x_{2}^{3}
\end{array}\right) \\
f_{2}(x)=\left(\begin{array}{l}
x_{2}+x_{1}^{2}-x_{1}^{3} \\
4 x_{1}+2 x_{2}
\end{array}\right)
\end{array}\right.
$$

The objective is to define a hybrid limit cycle for this system, which remains as close as possible to the desired point $x_{d}$, as well as to find the most rapid trajectory for reaching this limit cycle from every initial state that meets operating constraints (global operating domain and global reachability domain).

This system (6) belongs to the class defined by the $\operatorname{SDC}(3)$.

It should be noted that mode $l_{1}$ is stable at the origin ("spiral" trajectories) while mode $l_{2}$ is unstable (hyperbolic trajectories).

Now, let us determine the set of points around which a stable hybrid limit cycle can be designed for this system. $E=\left\{z \in \mathbb{R}^{2} / \operatorname{det}\left(f_{1}(z), f_{2}(z)\right)=0\right.$ and $\left\langle f_{1}(z) \mid f_{2}(z)\right\rangle<0$ and $p(z)$ is even $\}$

$$
\left\{\begin{array}{l}
\operatorname{det}\left(f_{1}(z), f_{2}(z)\right)=0 \Leftrightarrow-8 z_{1}^{2}-30 z_{1} z_{2}+ \\
2 z_{1}^{4}-6 z_{1}^{3} z_{2}-7 z_{2}^{2}-4 z_{2}^{3} z_{1}-z_{2}^{4}-6 z_{1}^{3}-z_{1}^{5}+ \\
3 z_{1}^{2} z_{2}+z_{1}^{2} z_{2}^{3}+z_{1}^{6}-z_{1}^{3} z_{2}^{3}=0 . \\
\left\langle f_{1}(z) \mid f_{2}(z)\right\rangle<0 \\
\Leftrightarrow\left(-2 z_{1}-z_{1}^{3}-5 z_{2}-z_{2}^{3}\right)\left(z_{2}+z_{1}^{2}-z_{1}^{3}\right)+ \\
\left(6 z_{1}+z_{1}^{3}-3 z_{2}-z_{2}^{3}\right)\left(4 z_{1}+2 z_{2}\right)<0 .
\end{array}\right.
$$

Figure 12 gives the dynamics of the two modes of the SDS, associated with vector field $f_{1}(x)$ (black stable "spiral" trajectories) and $f_{2}(x)$ (red unstable hyperbolic trajectories) and the set $\mathrm{E}$ (blue curves match the condition $\operatorname{det}\left(f_{1}(z), f_{2}(z)\right)=0$, and green curves match the condition $\left.\left\langle f_{1}(z) \mid f_{2}(z)\right\rangle=0\right)$. The properties of the $E$ points given in theorems 3.1 and 3.2 can be checked intuitively. Indeed, the properties $\operatorname{det}\left(f_{1}(z), f_{2}(z)\right)=0$ and $\left\langle f_{1}(z) \mid f_{2}(z)\right\rangle<0$ ensure that a limit cycle exists around each point of $E$.

Let $x_{d}=\left[\begin{array}{ll}-1 & 5\end{array}\right]^{T} \notin E$. The nearest point in $E$ such that $d\left(x_{d E}, x_{d}\right)=\min _{z \in E} d\left(z, x_{d}\right)$, is $x_{d E}=\left[\begin{array}{ll}-0.89 & 5.01\end{array}\right]^{T} \in E$. The hybrid limit cycle $C C\left(x_{c 1}, x_{c 2}\right)$ is determined by choosing one of the switching points, for example $x_{c 2}=\left[\begin{array}{ll}-0.5 & 5.5\end{array}\right]^{T}$. The second one can be calculated from the trajectories using the Lipchitz subsystems vector fields starting from $x_{c_{2}}$. The solution is unique for the both modes $f_{1}(x)$ and $f_{2}(x)$. The limit cycle $C C\left(x_{c 1}, x_{c 2}\right)$ passing through the switching point $x_{c 2}=\left[\begin{array}{ll}-0.5 & 5.5\end{array}\right]^{T}$ has $x_{c 1}=\left[\begin{array}{lll}-1.29 & 4.71\end{array}\right]^{T}$ as the second switching point. This hybrid limit cycle $C C\left(x_{c 1}, x_{c 2}\right)$ is given in figure 13 . Then, the 

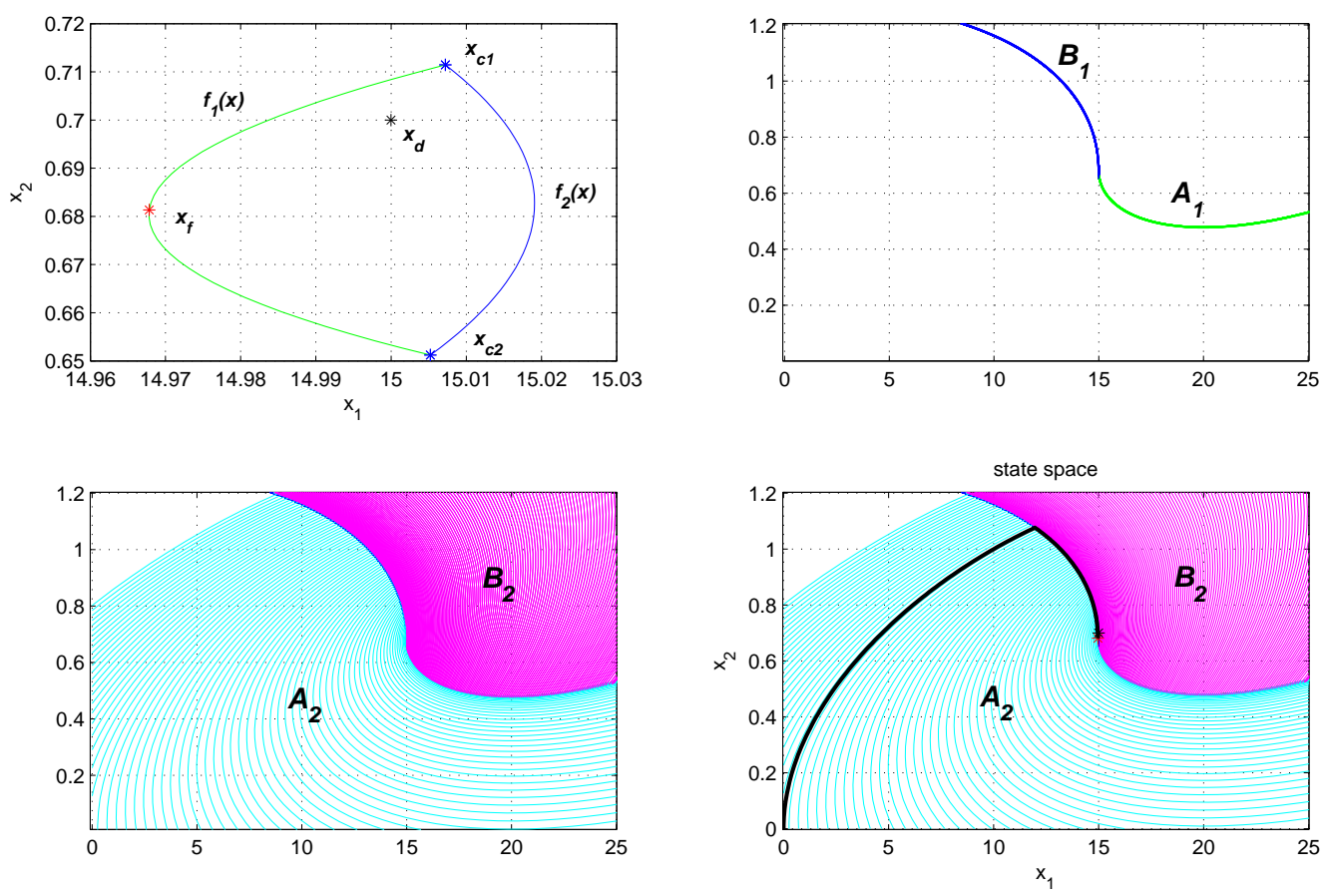

Figure 11: Synthesis and simulation results for the Buck converter.

global reachability domain $D$ can be determined by backward inference from the hybrid limit cycle $C C\left(x_{c 1}, x_{c 2}\right)$ (see definition 4.1 ) with respect to the global operating domain of the system, $\Omega$. $\Omega=\left\{x \in \mathbb{R}^{2} /-2 \leq x_{1} \leq 2\right.$ and $\left.-2 \leq x_{2} \leq 8\right\}$. All regions $A_{i}$ and $B_{i}$ are calculated until $A_{N+1}=B_{N}$. Thus, $D=\left(\bigcup_{i>1} A_{i}\right) \bigcup\left(\bigcup_{i>1} B_{i}\right)=A_{N+1}=B_{N}$. Figure 14 presents all $A_{i}$ 's and $B_{i}$ 's regions, $\Omega$ and $D$. Note that $A_{i}$ and $B_{i}$ regions expand step-by-step $\left(A_{i-1} \subset A_{i}\right.$ and $\left.B_{i-1} \subset B_{i}\right)$. According to the theorem 4.1, the SDS (6) is controlable for all $x_{0} \in D$, and there exists a switching sequence which drives the system from the initial state $x_{0}$ to the final hybrid limit cycle $C C\left(x_{c 1}, x_{c 2}\right)$. The possible switching sequences are: $\left(l_{1}, l_{2}\right)^{k},\left(l_{1}, l_{2}\right)^{k} l_{1},\left(l_{2}, l_{1}\right)^{k}$ and $\left(l_{2}, l_{1}\right)^{k} l_{2}$ with $k \in \mathbb{N}$.

The optimization of the constrained timed switching sequence to reach $C C\left(x_{c 1}, x_{c 2}\right)$ from different initial points of $D$ is done using time criterion and con- straints imposed by $\Omega$. The trajectory reaches the hybrid limit cycle at $x_{f}=\left[\begin{array}{ll}-1.2 & 4.8\end{array}\right]^{T} \in \Phi_{1}\left(x_{c 2}, \delta\right)$ with $0<\delta<\delta_{c 1}$. There are three examples of trajectories which drive the SDS from an initial point in $D$ to the hybrid limit cycle $C C\left(x_{c 1}, x_{c 2}\right)$ with a minimum total duration time:

- if $x_{0}=\left[\begin{array}{ll}-1.5 & 5\end{array}\right]^{T}$, the switching sequence is $\left(l_{1}, l_{2}\right)$, the duration times are $\left(\delta_{s_{1}}=\right.$ $\left.2.97 \mathrm{~ms}, \delta_{s_{2}}=63.89 \mathrm{~ms}\right)$ and the total reachability time is $t c=66.86 \mathrm{~ms}$ (pink trajectory).

- if $x_{0}=\left[\begin{array}{ll}2 & 1\end{array}\right]^{T}$, the switching sequence is $\left(l_{1}, l_{2}, l_{1}, l_{2}, l_{1}, l_{2}, l_{1}\right)$, the duration times are $\left(\delta_{s_{1}}=2,97 \mathrm{~ms}, \delta_{s_{2}}=344,66 \mathrm{~ms}, \delta_{s_{3}}=\right.$ $0,78 \mathrm{~ms}, \delta_{s_{4}}=61,13 \mathrm{~ms}, \delta_{s_{5}}=0.61 \mathrm{~ms}, \delta_{s_{6}}=$ $\left.53,54 \mathrm{~ms}, \delta_{s_{7}}=12.35 \mathrm{~ms}\right)$ and the total reachability time is $t c=476.02 \mathrm{~ms}$ (black trajectory).

- if $x_{0}=\left[\begin{array}{ll}-1 & 3\end{array}\right]^{T}$, the switching sequence is $\left(l_{2}, l_{1}\right)$, the duration times are $\left(\delta_{s_{1}}=464.36 \mathrm{~ms}, \delta_{s_{2}}=\right.$ 

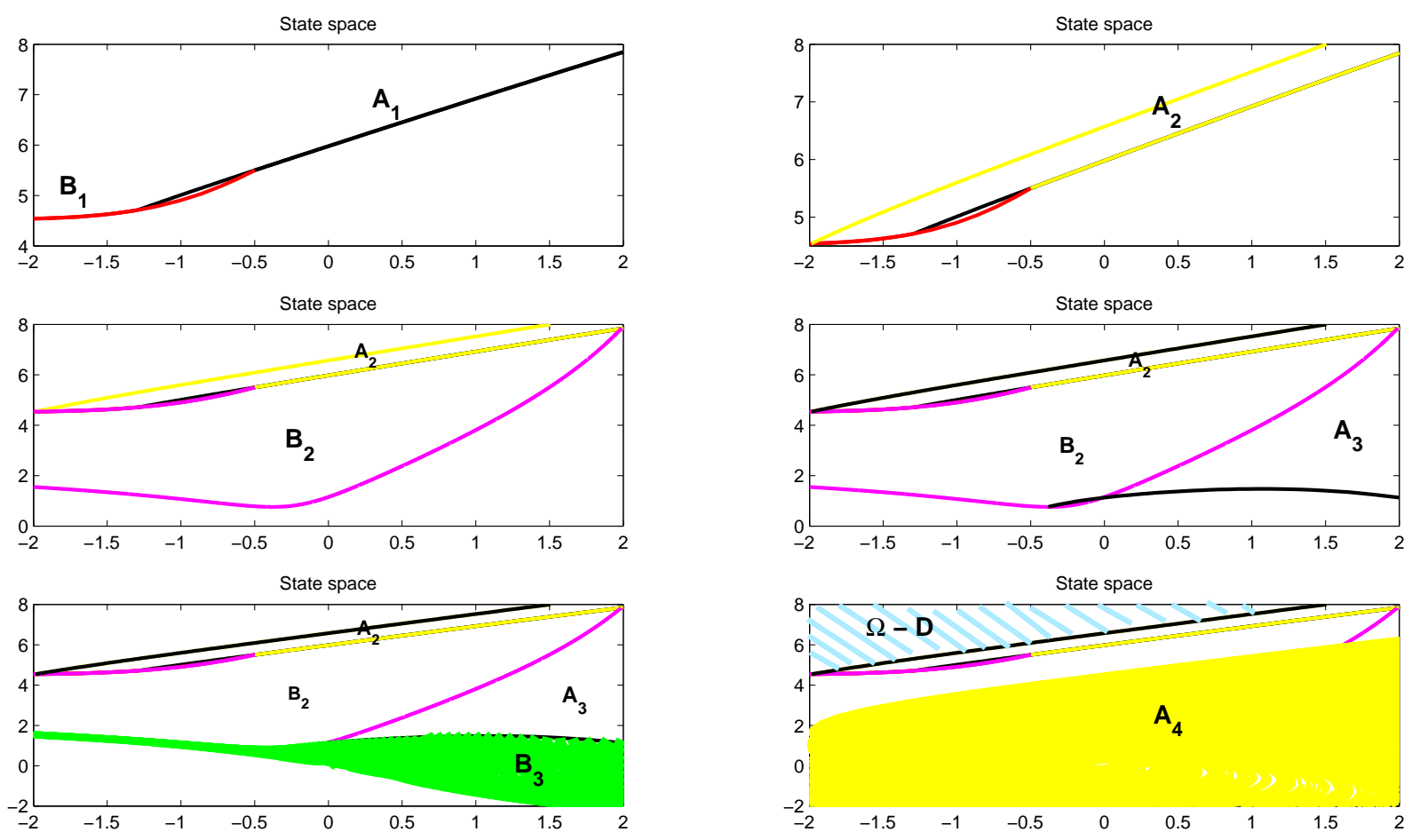

Figure 14: Synthesis results of the hybrid limit cycle and of the reachability domain, $D$, for the nonlinear example (6).

$10.61 \mathrm{~ms}$ ) and the total reachability time is $t c=$ extension is restricted.

$474.97 \mathrm{~ms}$ (blue trajectory).

Note that no shorter sequence can reach the hybrid limit cycle from these initial states, whilst meeting the constraints imposed by $\Omega$, and note that any longer sequence results a longer total reachability time.

The trajectories of the controlled SDS from its initial state to the chosen hybrid limit cycle are given in figure 15 .

To extend the theorems 3.1 and 3.2 to $\mathbb{R}^{n}$, the intention is to find a submanifold of $\mathbb{R}^{n}$ which is a surface and which includes the hybrid limit cycle $C C\left(x_{c 1}, x_{c 2}\right)$. Thus, the proofs of the theorems 3.1 and 3.2 extended to $\mathbb{R}^{n}$, can be carried out on this submanifold with dimension 2. As this submanifold must exist, the class of systems concerned by this

\section{Extension of the theorem 3.1 to $\mathbb{R}^{n}$}

Let us consider a SDS with the two following vector fields: $\forall i \in\{1,2\}, \dot{x}=f_{i}(x)=$ $\left[f_{i}^{1}(x) f_{i}^{2}(x), \cdots, f_{i}^{n}(x)\right]^{T}$ with $x \in \mathbb{R}^{n}$.

Let us consider the function $\varphi$ : $\varphi: \mathbb{R}^{n} \rightarrow \mathbb{R}^{n-2}$

$$
x \longmapsto \varphi(x)=\left[\varphi_{1}(x), \varphi_{2}(x), \cdots, \varphi_{n-2}(x)\right]^{T}
$$

Definition 9.1. $b \in \mathbb{R}^{n-2}$ is a regular value of $\varphi$ if and only if $\forall a \in \varphi^{-1}(b), \operatorname{rank}\left(J_{\varphi}(a)\right)=n-2$, with

$$
J_{\varphi}(a)=\left(\begin{array}{ccc}
\frac{\partial \varphi_{1}}{\partial x_{1}}(a) & \cdots & \frac{\partial \varphi_{1}}{\partial x_{n}}(a) \\
\vdots & & \vdots \\
\frac{\partial \varphi_{n-2}}{\partial x_{1}}(a) & \cdots & \frac{\partial \varphi_{n-2}}{\partial x_{n}}(a)
\end{array}\right)
$$




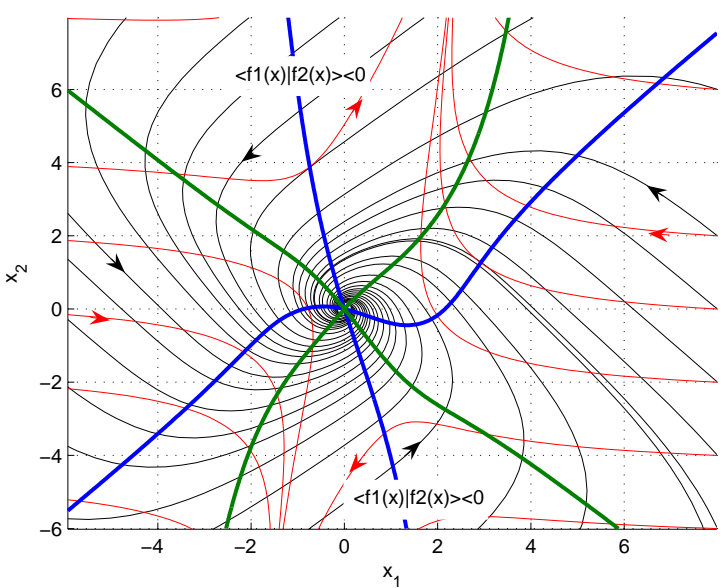

Figure 12: 2 Subsystems dynamics (red and black trajectories) and set $E$ (left-upper and right-lower parts of the blue curves) for the nonlinear example (6).

being the Jacobian matrix of $\varphi$ at a.

i.e. $b$ is not a stationary value of $\varphi$ and there exists a submatrix of $J_{\varphi}(a)$ of order $n$-2 which has a non-zero determinant.

In the sequel, we note:

- $\mathcal{M}=\varphi^{-1}(b)=\left\{x \in \mathbb{R}^{n} / \varphi(x)=b\right\}$ a submanifold of $\mathbb{R}^{n}$ with dimension 2 .

- $\mathcal{E}=\left\{z \in \mathbb{R}^{n} / \exists \eta<0 /\left(f_{1}(z)=\eta f_{2}(z)\right.\right.$ and $p(z)$ is even $\}$. In the plane, $\mathcal{E}$ represents $E$, the set of points with collinear and opposite direction $f_{1}(x)$ and $f_{2}(x)$ and non transverse trajectories.

- $T_{a} \mathcal{M}=\operatorname{ker}\left(J_{\varphi}(a)\right)$ is the set of vector fields which are tangent to the submanifold $\mathcal{M}$ at point a. Then, $T_{a} \mathcal{M}$ is orthogonal to $\operatorname{grad}\left(\varphi_{j}(a)\right)$, $j \in\{1, \cdots, n-2\}$.

- $\widetilde{\operatorname{Int}}\left(C C\left(x_{c_{1}}, x_{c_{2}}\right)\right)=\operatorname{Int}\left(C C\left(x_{c_{1}}, x_{c_{2}}\right) \bigcap \mathcal{M}\right)$ is the union of open sets in $\mathcal{M}$ bounded by the hybrid limit cycle $C C\left(x_{c_{1}}, x_{c_{2}}\right)$.

Theorem 9.1. Let us consider a SDS (3). Let $C C\left(x_{c 1}, x_{c 2}\right)$ in $\mathbb{R}^{n}$ be a hybrid limit cycle with $\left\{x_{c_{1}}, x_{c_{2}}\right\} \in \mathcal{M}, x_{c 1} \neq x_{c 2}$, with at least one point $x \in C C\left(x_{c 1}, x_{c 2}\right)$ such that $f_{1}(x) \neq-f_{2}(x)$. Let us

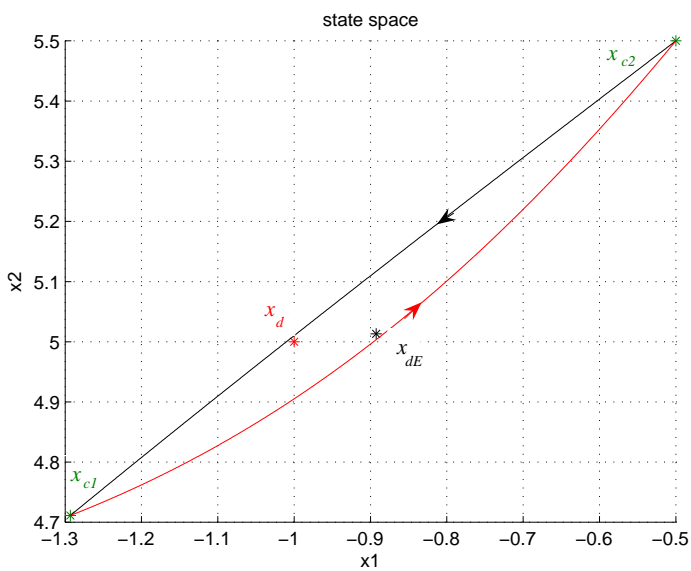

Figure 13: hybrid limit cycle of the nonlinear example (6).

consider $x_{0} \in \mathcal{E} \cap \mathcal{M}(\neq \phi)$ and $\gamma \in R_{+}$. If $\forall x \in$ $\mathcal{B}\left(x_{0}, \gamma\right) \cap \mathcal{M}, \forall i \in\{1,2\}$ and $\forall j \in\{1, \cdots, n-2\}$, $\left\langle f_{i}(x) \mid \operatorname{grad}\left(\varphi_{j}(x)\right)\right\rangle=0$, and if the closure of its interior, $\overline{\operatorname{Int}}\left(C C\left(x_{c 1}, x_{c 2}\right)\right)$, does not include any of the equilibrium points of the two dynamics $f_{1}$ and $f_{2}$, then, there exists a non-empty set of points $z \in \mathbb{R}^{n}$, such that $z \in \mathcal{E} \cap \overline{\overline{\operatorname{Int}}\left(C C\left(x_{c 1}, x_{c 2}\right)\right)} \subset \mathcal{B}\left(x_{0}, \gamma\right) \cap \mathcal{M}$ i.e. $\mathcal{E} \cap \overline{\widetilde{\operatorname{Int}}\left(C C\left(x_{c 1}, x_{c 2}\right)\right)} \neq \phi$.

Proof: As $\forall i \in\{1,2\}$ and $\forall j \in\{1, \cdots, n-2\}$, $\left\langle f\left(x, l_{i}\right) \mid \operatorname{grad}\left(\varphi_{j}(x)\right)\right\rangle=0, \forall x \in \mathcal{B}\left(x_{0}, \gamma\right) \cap \mathcal{M}$, the trajectory $x(t)$ stays inside $\mathcal{B}\left(x_{0}, \gamma\right) \cap \mathcal{M}$, whatever its length. Then, if $C C\left(x_{c 1}, x_{c 2}\right)$ exists, necessarily it is on $\mathcal{B}\left(x_{0}, \gamma\right) \cap \mathcal{M}$. Moreover, if $\widetilde{\widetilde{\operatorname{Int}}\left(C C\left(x_{c 1}, x_{c 2}\right)\right)}$ does not include any of the equilibrium points of the two dynamics $f_{1}$ and $f_{2}$, the proof of theorem 9.1 is equivalent to that of theorem 3.1 to conclude that $\mathcal{E} \cap \overline{\widetilde{\operatorname{Int}}}\left(C C\left(x_{c 1}, x_{c 2}\right)\right) \neq \phi$.

\section{Extension of the theorem 3.2 to $\mathbb{R}^{n}$}

Theorem 10.1. Let us consider $x_{0} \in \mathcal{E} \cap \mathcal{M}(\neq \phi)$ and $\gamma>0$. If $\forall x \in \mathcal{B}\left(x_{0}, \gamma\right) \cap \mathcal{M}, \forall i \in\{1,2\}, \forall j \in$ $\{1, \cdots, n-2\},\left\langle f_{i}(x) \mid \operatorname{grad}\left(\varphi_{j}(x)\right)\right\rangle=0$, a hybrid limit cycle $C C\left(x_{c 1}, x_{c 2}\right)$ exists around the point $x_{0}$ (such that $\left.x_{0} \in \overline{\overline{\operatorname{Int}}}\left(C C\left(x_{c 1}, x_{c 2}\right)\right)\right)$. 


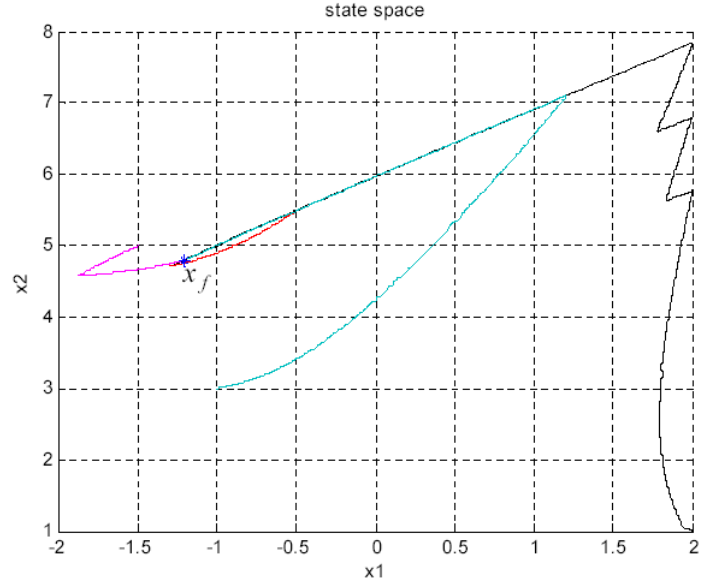

Figure 15: Trajectories of the controlled SDS (6) to reach the hybrid limit cycle from different initial points

Proof: The proof of theorem 10.1 is the same as the proof of theorem 3.2 because $\mathcal{E} \cap \mathcal{M}$ is a submanifold of $\mathbb{R}^{n}$ and $\mathcal{M}$ is a surface which includes the hybrid limit cycle $C C\left(x_{c 1}, x_{c 2}\right)$.

Then, the existence of a hybrid limit cycle for the switched dynamical system is directly related to the existence of the submanifold $\mathcal{M} \neq \phi$ which is defined from a regular point of the function $\varphi$. The submanifold $\mathcal{M}$ is the surface which includes the hybrid limit cycle. If the SDS is switching between two discrete modes with continuous dynamics in $\mathbb{R}^{2}$, this surface $\mathcal{M}$ is the plane $\mathbb{R}^{2}$.

\section{Nonlinear example in $\mathbb{R}^{3}$}

Let us consider two nonlinear three dimensional vector fields to illustrate theorems 9.1 and 10.1:

$$
\begin{gathered}
f_{1}(x)=\left(\begin{array}{l}
x_{1} x_{2}^{2} \\
-x_{1}^{2} x_{2}+x_{3} \\
-x_{2}
\end{array}\right) \\
f_{2}(x)=\left(\begin{array}{l}
x_{2}\left(x_{1}^{2}+x_{2}^{2}+x_{3}^{2}\right) \\
-x_{1}+x_{3}^{2} \\
-x_{2} x_{3}
\end{array}\right) .
\end{gathered}
$$

Let us consider the function $\varphi$ :

$$
\begin{aligned}
& \varphi: \quad \mathbb{R}^{3} \rightarrow \mathbb{R} \\
& \quad\left(x_{1} x_{2} x_{3}\right) \longmapsto x_{1}^{2}+x_{2}^{2}+x_{3}^{2}
\end{aligned}
$$

$\forall b \in \mathbb{R}_{+}, \forall a \in \varphi^{-1}(b), \operatorname{rank}\left(J_{\varphi}(a)\right)=1$. Then, $b$ is a regular value of $\varphi$.

The submanifold in $\mathbb{R}^{3}, \mathcal{M}=\varphi^{-1}(b)$, is the sphere:

$$
\mathcal{M}=\left\{x \in \mathbb{R}^{3} / x_{1}^{2}+x_{2}^{2}+x_{3}^{2}=b, b \in \mathbb{R}_{+}\right\}
$$

$\mathcal{E}$ is the set of points in $\mathbb{R}^{3}$ such that $f_{1}(x)=\eta f_{2}(x)$, with $\eta<0$ and $p(z)$ is even.

$$
\mathcal{E}=\left\{x \in \mathbb{R}^{3} / x_{1} x_{2} x_{3}=1\right\}
$$

Choosing $\mathrm{b}=6$ ensures that $\mathcal{E} \cap \mathcal{M} \neq \phi$. A quadrant of the submanifolds $\mathcal{M}$ and $\mathcal{E}$ and of their intersections is given in figure 16 .

Since $\forall i \in\{1,2\}, \forall x \in \mathcal{M},\left\langle f_{i}(x) \mid \operatorname{grad}(\varphi(x))\right\rangle=$ 0 , then, for $x_{0} \in \mathcal{E} \cap \mathcal{M}(\neq \phi)$ and $\gamma>0, \forall x \in$ $\mathcal{B}\left(x_{0}, \gamma\right) \cap \mathcal{M},\left\langle f_{i}(x) \mid \operatorname{grad}(\varphi(x))\right\rangle=0$ and a hybrid limit cycle $C C\left(x_{c 1}, x_{c 2}\right)$ exists around the point $x_{0}$.
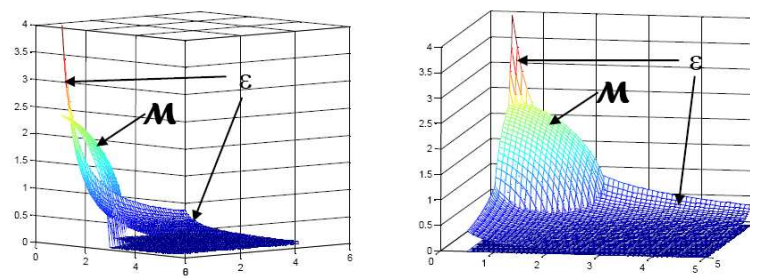

Figure 16: Submanifolds $\mathcal{M}$ and $\mathcal{E}$ of nonlinear example with vector fields (7) and (8) in $\mathbb{R}^{3}$.

\section{Conclusion}

This paper presents a new constructive method for the synthesis of a stabilizing control for a class of switched dynamical systems in $\mathbb{R}^{2}$, switching between two discrete modes, without state discontinuity and which respect the technological constraints (minimum duration between two successive switchings, boundedness of the real valued state variables). For each mode, the system dynamic is continuous, linear or nonlinear. The main result of this paper, a necessary and sufficient condition of existence and 
stability of a hybrid limit cycle consisting of a sequence of two operating modes in $\mathbb{R}^{2}$, is presented in theorems 3.1 and 3.2. The method is based on a geometric approach. It is made of three main steps: firstly, determining a stable hybrid limit cycle around the desired operating point, $x_{d}$ or as close as possible to it, then calculating the regions of the state space from which the hybrid limit cycle is reachable from the initial state and finally synthesizing a stabilizing control which optimizes time. Theorems 9.1 and 10.1 extend to $\mathbb{R}^{n}, n>2$ the necessary and sufficient condition of existence and stability of a hybrid limit cycle proven in $\mathbb{R}^{2}$ but only for a restricted class of systems. Then, the stabilizing control synthesis method can be extended to $\mathbb{R}^{n}, n>2$. Different examples are used to illustrate these theorems.

\section{Acknowledgments}

The authors would like to thank the French Ministry of Foreign and European Affairs and the Tunisian Ministry of Higher Eduction, Scientific Research and Technology for their financial support for the project PHC-Utique 09G 1413 managed by CMCU. They would also like to thank the CNRS/SEE working group on Hybrid Dynamical Systems (HDS) and the GDR MACS for the enlightening scientific discussions about HDS.

\section{References}

[1] J. Ben Salah, H. Jerbi, C. Valentin, C. Z. Xu, "Synthèse géométrique du cycle limite hybride optimal et de la commande d'une classe de systèmes dynamiques à commutation", e-STA, vol. 5, issue 4, 2008, pp 33-41.

[2] J. Ben Salah, C. Valentin, H. Jerbi. Quadratic Common Lyapunov Fonction computation and planar linear switched system stabilization, in ECC'09, European Control Conference, Budapest, Hungary, 6 pages, 23-26 August 2009.

[3] O. Bethoux, J-P. Barbot, "Commande permettant le contrôle du convertisseur multicellulaire série à nombre non premier de cellules", in $e$ $S T A$, vol. 4, 2007, pp. 44-49.
[4] U. Boscain, G. Charlot, M. Sigalotti, "Stability of Planar Nonlinear Switched Systems", , $D C D S-A$, Vol 15, n.2, June 2006, pp. 415-432.

[5] U. Boscain, "A review on stability of switched systems for arbitrary switchings", in Proceedings of the conference "Geometric Control and Nonsmooth Analysis", Rome, Italy, June 5 - 9, 2006, "Series on Advances in Mathematics for Applied Sciences", Worldscientific, pp. 100-119, 2007.

[6] R. Bourdais, L. Hetel, J. Daafouz, W. Perruquetti, "Stabilité et stabilisation d'une classe de systèmes dynamiques hybrides", Journal Européen des Systèmes Automatisés, vol. 41, issue 7-8/2007, pp. 819-853.

[7] M.S. Branicky, "Stability of switched and Hybrid systems", in 33rd Proceeding IEEE Conference on Decision and Control, vol. 4, Lake Buena Vista FL USA, 1994, pp. 3498-3503.

[8] M. S. Branicky, "Multiple Lyapunov functions and other analysis tools for switched and hybrid systems", in IEEE Transaction on Automatic Control, Vol. 43, Issue 4, April 1998, pp. $475-482$.

[9] R.W. Brockett, "Asymptotic Stability and Feedback Stabilization", in Differential Geometrics in Control Theory (R. W. Brockett et al., eds.), Progress in Mathematics, Vol. 27. Birkhauser Publishers, Boston, MA: 1983, pp. 181-191.

[10] S.Chaib, D. Boutat, A. Benali, F. Kratz, "Dynamic control of switched linear systems: a commun Lyapunov function approach", in 45th Proceeding IEEE Conference on Decision and Control, San Diego CA USA, December 2006.

[11] J. Daafouz, P. Riedinger, C. Iung, "Static Output Feedback Control for switched systems" in IEEE Conference on Decision and Control, Orlando, Florida, USA, December 4-7, 2001.

[12] J. Goncalves, "Constructive Global Analysis of Hybrid Systems", Ph.D. Thesis, Massachusetts Institute of Technology, September 2000. 
[13] H. Gueguen, M.A. Lefebvre, J. Zaytoon, O. Nasri, "Safety verification and reachability analysis for hybrid systems", in Annual Reviews in Control, vol. 33, 2009, pp. 2536.

[14] M. Johansson, A. Rantzer, "Computation of piecewise quadratic Lyapunov fonctions for hybrid systems", in IEEE Transaction on Automatic Control, Vol. 43, Issue 4, Apr 1998, pp 555-559.

[15] D. Liberzon and A. S. Morse, "Basic problems in stability and design of switched systems", IEEE Control Systems Magazine, 19 (1999), pp. 5970.

[16] D. Liberzon, "Switching in Systems and Control", Volume in series Systems and Control: Foundations and Applications. ISBN 0-81764297-8, Birkhauser Boston, Jun 2003.

[17] P. Manon, "Sur l'optimisation des séquences de fonctionnement des systèmes dynamiques hybrides", Ph.D. Thesis, Université Claude Bernard Lyon1, Mars 2001.

[18] P. Manon, C. Valentin-Roubinet, G. Gilles, "Optimal Control of Hybrid Dynamical Systems: Application in Process Engineering", in Control Engineering Practice, vol. 10, 2002, pp. 133-149.

[19] M. Margaliot, "Stability analysis of switched systems using variational principles: An introduction", Automatica, vol. 42, pp. 2059.2077, 2006.

[20] S. Pettersson , B. Lennartson, "A LMI approach for stability analysis of nonlinear systems", in Proceeding of EEC, Brussel, Belgique, juillet 1997.

[21] S. Pettersson, "Synthesis of Switched Linear Systems", in 42nd Proceeding IEEE Conference on Decision and Control, Maui, Hawaii USA, December 2003.

[22] D. Pinon, M. Fadel, T. Meynard, "Commande par mode glissant d'un hacheur à deux cellules : étude de l'installation des cycles limites", Revue Internationale de Génie Electrique, vol. 1, n 3, 1998, pp. 393-415.
[23] M. Rubensson, "Stability Properties of Switched Dynamical Systems A Linear Matrix Inequality Approach", Ph.D. Thesis, Goteborg : Chalmers University of Technology, April 2003.

[24] S. N. Simic, K.H. Johansson, J. Lygeros, and S. Sastry. "Hybrid limit cycles and hybrid Poincare-Bendixson"' . in Proceeding of IFAC World Congress, Barcelona, Spain, July 22-26 2002.

[25] A.J. Van der Schaft, H. Schumacher, "An introduction to hybrid dynamical systems", ed Springer-Verlag, Great Britain, 2000.

[26] X. Xu, P. J. Antsaklis, "Design of stabilizing control laws for second-order switched systems", in 14th Proceeding of IFAC World Congress, Vol. C, Beijing, China, July 1999, pp. 181-186.

[27] M. Zorn, "A remark on method in transfinite algebra", in Bull. Amer. Math. Soc, 41, 1935, pp 667-670. 